Proyecciones Journal of Mathematics

Vol. 36, No 4, pp. 727-737, December 2017.

Universidad Católica del Norte

Antofagasta - Chile

\title{
Existence of solutions for a nonlinear fractional system with nonlocal boundary conditions
}

\author{
A. Guezane-Lakoud \\ University Badji Mokhtar Annaba, Algeria \\ G. Rebiai \\ University of Guelma, Algeria and \\ R. Khaldi \\ University Badji Mokhtar Annaba, Algeria \\ Received : March 201\%. Accepted : April 2017
}

\begin{abstract}
In this paper, we use fixed point theorems to prove the existence and uniqueness of solution for a nonlinear fractional system with boundary conditions. At the end we present two examples illustrating the obtained results.
\end{abstract}

Keywords: Fractional Rieman-Liouville derivative, Fractional differential equation, Fixed Point Theorem.

Mathematics Subject Classification: 34B10, $26 A 33,34 B 15$. 


\section{Introduction}

In recent years, the theory of differential fractional equations has become an interesting field to explore. It is to be noted that such theory has many applications in several events existing in the real world, and also in many sciences such as: engineering, physics, chemistry, biology, etc ..., [13]. Moreover, the study of the systems of fractional differential equations has become more and more popular tool for controlling and modeling different systems $[2,7,15-17]$. Thus the fixed point theory is a powerful mathematical tool in the study of the existence, uniqueness, positivity and stability of solutions, see [1,3-6,9-14].

In this work, we consider the following system of fractional differential equations with boundary conditions:

$$
(F S)\left\{\begin{array}{c}
-D_{0^{+}}^{\alpha} u(t)=g(t) f(u(t)), 0<t<1, \\
u(0)=u^{\prime}(0)=0, a u(1)+b u^{\prime}(1)=0,
\end{array}\right.
$$

where $D_{0^{+}}^{\alpha}$ denotes the Reimann-Liouville fractional derivative, $2<\alpha<3$, $u=\left(u_{1}, u_{2}, \ldots, u_{n}\right)^{T}$ is an unknown function with

$u_{i}:[0,1] \rightarrow \mathbf{R}, g:[0,1] \rightarrow \mathbf{R}$ is a given function, $f: \mathbf{R}^{n} \rightarrow \mathbf{R}^{n}$, $f(u)=\left(f_{1}\left(u_{1}, u_{2}, \ldots, u_{n}\right), \ldots, f_{n}\left(u_{1}, u_{2}, \ldots, u_{n}\right)\right)^{T}, f_{i}: \mathbf{R}^{n} \rightarrow \mathbf{R}$.

This paper is organized as follows: in Section 2, some preliminary materials to be used later are stated. In Section 3, we present and prove our main results consisting of the existence and uniqueness of the solution of (FS). Finally our study is ended by an example illustrating the obtained results.

\section{Preliminaries}

In this section, we recall the basic definitions and lemmas from the fractional calculus theory, see [13].

Definition 1. The Riemann-Liouville fractional integrals of order $\alpha$ of a function $h$ is defined as

$$
I_{a^{+}}^{\alpha} h(t)=\frac{1}{\Gamma(\alpha)} \int_{a}^{t} \frac{h(s)}{(t-s)^{1-\alpha}} d s .
$$

Definition 2. The Riemann-Liouville derivative of fractional order $\alpha>0$ for a function $h$ is defined as

$$
D_{0^{+}}^{\alpha} h(t)=\frac{1}{\Gamma(n-\alpha)}\left(\frac{d}{d t}\right)^{n} \int_{0}^{t}(t-s)^{n-\alpha-1} h(s) d s=\left(\frac{d}{d t}\right)^{n} I^{n-\alpha} h(t),
$$

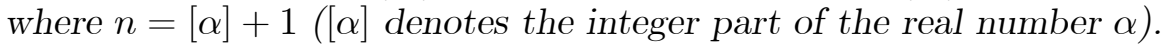


Lemma 3. For $\alpha>0$, the general solution of the homogeneous equation $D_{0^{+}}^{\alpha} u(t)=0$, is given by

$$
u(t)=c_{0} t^{\alpha-n}+c_{1} t^{\alpha-n-1}++c_{n-2} t^{\alpha-2}+c_{n-1} t^{\alpha-1},
$$

where $c_{i}, i=1,2, \ldots, n-1$, are arbitrary real constants.

Lemma 4. Let $p, q \geq 0, f \in L_{1}[a, b]$. Then

$$
I_{0^{+}}^{p} I_{0^{+}}^{q} f(t)=I_{0^{+}}^{p+q} f(t)=I_{0^{+}}^{q} I_{0^{+}}^{p} f(t) .
$$

\section{Main results}

Lemma 5. Let $y \in C([0,1], \mathbf{R})$. Assume that $a, b \in \mathbf{R}$ such that $a-$ $b(\alpha-1) \neq 0$, then for $i \in\{1, . ., n\}$, the linear nonhomogeneous problem

$$
\left(S_{i}\right)=\left\{\begin{array}{c}
-D_{0^{+}}^{\alpha} u_{i}(t)=y(t), 0<t<1 \\
u_{i}(0)=u_{i}^{\prime}(0)=0, a u_{i}(1)-b u_{i}^{\prime}(1)=0, i \in\{1, . ., n\}
\end{array}\right.
$$

has the following solution

$$
u_{i}(t)=\int_{0}^{1} G_{i}(t, s) y(s) d s, i \in\{1, . ., n\}
$$

where

$$
G_{i}(t, s)=\left\{\begin{array}{c}
-\frac{(t-s)^{\alpha-1}}{\Gamma(\alpha)}+ \\
\frac{t^{\alpha-1}}{a-b(\alpha-1)}\left(\frac{a}{\Gamma(\alpha)}(1-s)^{\alpha-1}-\frac{b}{\Gamma(\alpha-1)}(1-s)^{\alpha-2}\right), s \leq t \\
\frac{t^{\alpha-1}}{a-b(\alpha-1)}\left(\frac{a}{\Gamma(\alpha)}(1-s)^{\alpha-1}-\frac{b}{\Gamma(\alpha-1)}(1-s)^{\alpha-2}\right), s \geq t
\end{array}\right.
$$


Proof. Let $u_{i}$ be a solution of the fractional boundary value problem $(F S)$. Using Lemma 3, we obtain

$$
u_{i}(t)=-I_{0^{+}}^{\alpha} y(t)+A t^{\alpha-1}+B t^{\alpha-2}+C t^{\alpha-3},
$$

then, by multiplying (3.4) by $t^{3-\alpha}$, it yields

$$
\mathrm{t}^{3-\alpha} u_{i}(t)=-I_{0^{+}}^{\alpha} y(t) t^{\alpha-3}+A t^{2}+B t+C .
$$

According to the condition $u(0)=0$, we obtain $C=0$. Therefore, differentiating (3.4), we have

$$
u_{i}^{\prime}(t)=-I_{0^{+}}^{\alpha-1} y(t)+(\alpha-1) A t^{\alpha-2}+(\alpha-2) B .
$$

Multiplying (3.5) by $t^{3-\alpha}$, we obtain

$$
t^{3-\alpha} u_{i}^{\prime}(t)=-I_{0^{+}}^{\alpha-1} y(t) t^{3-\alpha}+(\alpha-1) A t+(\alpha-2) B .
$$

From condition $u_{i}^{\prime}(0)=0$, it follows $B=0$, thus,

$$
u_{i}(t)=-I_{0^{+}}^{\alpha} y(t)+A t^{\alpha-1} .
$$

Since $a u_{i}(1)-b u_{i}^{\prime}(1)=0$, then

$$
A=\frac{a}{a-b(\alpha-1)} I_{0^{+}}^{\alpha} y(1)-\frac{b}{a-b(\alpha-1)} I_{0^{+}}^{\alpha-1} y(1) .
$$

By substituting $A$ in (3.7), we get $\mathrm{u}_{i}(t)=\int_{0}^{1} G_{i}(t, s) y(s) d s$.

Lemma 6. If $a>0$ and $b<0$, then the functions $G_{i}$ are nonnegative, continuous

and

$$
G_{i}(t, s) \leq \frac{1}{\Gamma(\alpha-1)}, \forall s, t \in[0,1], i \in\{1, . ., n\}
$$

Proof. The proof is direct, we omit it.

Let $X$ be the Banach space of all functions $u \in C^{n}[0,1]=C[0,1] \times \ldots \times C[0,1]$ with the norm $\|$.$\| defined by \|u\|=\sum_{i=1}^{i=n} \max _{t \in[0,1]}\left|u_{i}(t)\right|$. Define the integral operator $T: X \rightarrow X$ by $T(u)=\left(T_{1} u, T_{2} u, \ldots, T_{n} u\right)$, where 


$$
\left(T_{i} u\right)(t)=\int_{0}^{1} G_{i}(t, s) g(s) f_{i}(u(s)) d s, i=1, \ldots, n .
$$

Lemma 7. The function $u \in X$ is a solution of the system $(F S)$ if and only if $T_{i} u(t)=u(t)$, for all $t \in[0,1], \forall i \in\{1, \ldots, n\}$.

The first main statement in this work is the uniqueness of solution of the boundary problem $(F S)$.

Theorem 8. Assume that

i) $f_{i} \in C\left(\mathbf{R}^{n}, \mathbf{R}\right), g \in L^{1}([0,1], \mathbf{R})$

ii) There exists a constant $L>0$ such that

$$
\left|f_{i}\left(x_{1}, . ., x_{n}\right)-f_{i}\left(y_{1}, \ldots, y_{n}\right)\right| \leq L \sum_{i=1}^{n}\left|x_{i}-y_{i}\right|
$$

and

$$
\frac{n L\|g\|_{L^{1}[0,1]}}{\Gamma(\alpha-1)}<1,
$$

for all $t \in[0,1]$ and for all $x_{i}, y_{i} \in \mathbf{R}, i=1, \ldots n$. Then, the boundary value problem $(F S)$ has a unique solution in $X$.

Proof. We will use the Banach contraction principle to prove that the operator $T$ has a fixed point. Using the properties of the function $G_{i}$, it yields

$$
\begin{aligned}
\left|T_{i} x(t)-T_{i} y(t)\right| & \leq \int_{0}^{1}\left|G_{i}(t, s)\right||g(s)|\left|f_{i}(x(s))-f_{i}(y(s))\right| d s \\
& \leq \frac{L}{\Gamma(\alpha-1)} \int_{0}^{1}|g(s)| \sum_{i=1}^{n}\left|x_{i}(s)-y_{i}(s)\right| d s \\
& \leq \frac{L}{\Gamma(\alpha-1)}\|g\|_{L^{1}[0,1]}\|x-y\|,
\end{aligned}
$$

then by taking the maximum over $t \in[0,1]$, it follows

$$
t \in[0,1] \max \left|T_{i} x(t)-T_{i} y(t)\right| \leq \frac{L}{\Gamma(\alpha-1)}\|g\|_{L^{1}[0,1]}\|x-y\|
$$


Summing the $n$ inequalities in (3.13), it yields

$$
\|T x-T y\| \leq \frac{n L\|g\|_{L^{1}[0,1]}}{\Gamma(\alpha-1)}\|x-y\| \text {. }
$$

Since $\frac{n L\|g\|_{L^{1}[0,1]}}{\Gamma(\alpha-1)}<1$, then $T$ is a contraction. As a consequence of Banach fixed-point theorem, we deduce that $T$ has a fixed point that is the unique solution of the (FS), this achieves the proof.

The second mains statement of this work is an existence result for the boundary problem $(F S)$.

Theorem 9. Assume that $f_{i}(0) \neq 0, i \in\{1, . ., n\}$, there exist $\eta>0$ and a nonnegative function $\Psi \in C\left(\mathbf{R}^{n},(0, \infty)\right)$ satisfying $\Psi\left(x_{1}, \ldots, x_{n}\right) \leq$ $\Psi\left(y_{1}, \ldots, y_{n}\right)$ for $0 \leq x_{i} \leq y_{i}, i=1, \ldots, n$. If

$$
\left|f_{i}(u)\right| \leq \Psi(|u|),
$$

for all $t \in[0,1]$ and all $u \in \mathbf{R}^{n}$ and

$$
\frac{n}{\Gamma(\alpha-1)} \Psi(\eta, \ldots, \eta)\|g\|_{L^{1}[0,1]} \leq \eta,
$$

then, the problem (FS) has at least one nontrivial solution $u^{*} \in X$.

For the proof of Theorem we need the nonlinear alternative of LeraySchauder:

Lemma 10. Let $F$ be a Banach space and $\Omega$ a bounded open subset of $F$, $0 \in \Omega$. Let $T: \Omega \rightarrow F$ be a completely continuous operator. Then, either there exists $x \in \partial \Omega, \lambda>1$ such that $T(x)=\lambda x$, or there exists a fixed point $x \in \Omega$ of $T$.

Proof. of Theorem 9. The continuity of the operator $T$ follows from the continuity of $f$. Set $B_{\eta}=\{u \in X:\|u\| \leq \eta\}$. Let us prove that $T: B_{\eta} \rightarrow X$ is a completely continuous operator. From (3.14), we have for each $t \in[0,1]$

$$
\begin{aligned}
\left|T_{i} u(t)\right| & \leq \int_{0}^{1}\left|G_{i}(t, s)\right||g(s)|\left|f_{i}(u(s))\right| d s \\
& \leq \frac{1}{\Gamma(\alpha-1)} \int_{0}^{1}|g(s)| \Psi(|u(s)|) d s
\end{aligned}
$$




$$
\begin{aligned}
& =\frac{1}{\Gamma(\alpha-1)} \int_{0}^{1}|g(s)| \Psi\left(\left|u_{1}(s)\right|, \ldots,\left|u_{2}(s)\right|\right) d s \\
& \leq \frac{1}{\Gamma(\alpha-1)} \Psi(\eta, \ldots, \eta)\|g\|_{L^{1}[0,1]}
\end{aligned}
$$

Taking the supremum over $[0,1]$, then summing the obtained inequalities according to $i$ from 1 to $n$, we get

$$
\|T u\| \leq \frac{n \Psi(\eta, \ldots, \eta)\|g\|_{L^{1}[0,1]}}{\Gamma(\alpha-1)}
$$

which implies that $T\left(B_{\eta}\right)$ is uniformly bounded.

Let us show that $(T u)$ is equicontinuous, $u \in B_{\eta}$. Let $t_{1}, t_{2} \in[0,1], t_{1}<t_{2}$, then

$$
\begin{aligned}
\left|T_{i} u\left(t_{1}\right)-T_{i} u\left(t_{2}\right)\right| \leq & \int_{0}^{1}\left|G_{i}\left(t_{1}, s\right)-G_{i}\left(t_{2}, s\right)\right||g(s)|\left|f_{i}(u(s))\right| d s \\
\leq & \int_{0}^{t_{1}}\left|G_{i}\left(t_{1}, s\right)-G_{i}\left(t_{2}, s\right)\right||g(s)|\left|f_{i}(u(s))\right| d s \\
& +\int_{t_{1}}^{t_{2}}\left|G_{i}\left(t_{1}, s\right)-G_{i}\left(t_{2}, s\right)\right||g(s)|\left|f_{i}(u(s))\right| d s \\
& +\int_{t_{2}}^{1}\left|G_{i}\left(t_{1}, s\right)-G_{i}\left(t_{2}, s\right)\right||g(s)|\left|f_{i}(u(s))\right| d s
\end{aligned}
$$

then

$$
\begin{aligned}
& \left|T_{i} u\left(t_{1}\right)-T_{i} u\left(t_{2}\right)\right| \leq \\
& \frac{\Psi(\eta, \ldots, \eta)}{\Gamma(\alpha)}\left[\int_{0}^{t_{1}}\left[\left(t_{2}^{\alpha-1}-t_{1}^{\alpha-1}\right)+\left(t_{2}-s\right)^{\alpha-1}-\left(t_{1}-s\right)^{\alpha-1}\right]|g(s)| d s\right. \\
& +\int_{t_{1}}^{t_{2}}\left[\left(t_{2}^{\alpha-1}-t_{1}^{\alpha-1}\right)+\left(t_{2}-s\right)^{\alpha-1}\right]|g(s)| d s \\
& \left.+\int_{t_{2}}^{1}\left[\left(t_{2}^{\alpha-1}-t_{1}^{\alpha-1}\right)\right]|g(s)| d s\right] \\
& \quad \leq \frac{\Psi(\eta, \ldots, \eta)}{\Gamma(\alpha)}\left[\left[\left(t_{2}^{\alpha-1}-t_{1}^{\alpha-1}\right)+\left(t_{2}-t_{1}\right)^{\alpha-1}\right] \int_{0}^{t_{1}}|g(s)| d s\right. \\
& \quad+\left[\left(t_{2}^{\alpha-1}-t_{1}^{\alpha-1}\right)+\left(t_{2}-t_{1}\right)^{\alpha-1}\right] \int_{t_{1}}^{t_{2}}|g(s)| d s
\end{aligned}
$$




$$
\begin{aligned}
& +\left[\left(t_{2}^{\alpha-1}-t_{1}^{\alpha-1}\right)\right] \int_{t_{2}}^{1}|g(s)| d s \\
\leq & \frac{\Psi(\eta, \ldots, \eta)}{\Gamma(\alpha)}\left[3\left(t_{2}^{\alpha-1}-t_{1}^{\alpha-1}\right)+2\left(t_{2}-t_{1}\right)^{\alpha-1}\right] \int_{0}^{1}|g(s)| d s .
\end{aligned}
$$

As $t_{1} \rightarrow t_{2}$, the right-hand side of the above inequality tends to zero. By Ascoli-Arzela theorem, we conclude that the operator $T: X \rightarrow X$ is completely continuous.

Now we apply the nonlinear alternative of Leray-Schauder. Let $u \in$ $\partial B_{\eta}$, such that $u=\lambda T u$ for some $0<\lambda<1$. We have

$$
\begin{aligned}
u_{i}(t) & =\lambda T_{i} u(t) \leq t \in[0,1] \max \left|T_{i} u(t)\right| \\
& \leq \frac{1}{\Gamma(\alpha-1)} \Psi(\eta, \ldots, \eta)\|g\|_{L^{1}[0,1]} .
\end{aligned}
$$

Taking the supremum over $[0,1]$, then summing the obtained inequalities according to $i$ from 1 to $n$, we get

$$
\|u\| \leq \frac{n}{\Gamma(\alpha-1)} \Psi(\eta, \ldots, \eta)\|g\|_{L^{1}[0,1]} .
$$

taking into account (3.15) we conclude

$$
\|u\|<\eta
$$

that contradicts the fact that $u \in \partial B_{\eta}$. So, we conclude that $T$ has at least one fixed point $u^{*} \in B_{\eta}$ and then the $(F S)$ has a nontrivial solution $u^{*} \in B_{\eta}$.

\section{Examples}

In this section, we give examples to illustrate the usefulness of our main results.

Example 1. Consider the following two-dimensional fractional order system

$$
\left(S_{i}\right)=\left\{\begin{array}{cc}
D_{0^{+}}^{\frac{5}{2}} u_{1}(t)=2 t \frac{e^{-\left(u_{1}^{2}+u_{2}^{2}\right)}}{1+u_{1}^{2}+u_{2}^{2}}, & D_{0^{+}}^{\frac{5}{2}} u_{2}(t)=2 t \frac{e^{-u_{1}^{2}}}{1+u_{1}^{2}+u_{2}^{2}} \\
u_{1}(0)=0, u_{1}^{\prime}(0)=0, & u_{2}(0)=0, u_{2}^{\prime}(0)=0 \\
a u_{1}(1)-b u_{1}^{\prime}(0)=0, & a u_{2}(1)-b u_{2}^{\prime}(0)=0
\end{array}\right.
$$


We have $\alpha=\frac{5}{2}, g(t)=2 t, f_{1}\left(u_{1}, u_{2}\right)=\frac{e^{-\left(u_{1}^{2}+u_{2}^{2}\right)}}{1+u_{1}^{2}+u_{2}^{2}}, f_{2}\left(u_{1}, u_{2}\right)=\frac{e^{-u_{1}^{2}}}{1+u_{1}^{2}+u_{2}^{2}}$, $f_{i} \in C\left(\mathbf{R}^{2}, \mathbf{R}\right), f_{i}(0) \neq 0$. If we choose $\Psi\left(u_{1}, u_{2}\right)=\frac{1}{1+u_{1}^{2}+u_{2}^{2}}$, then

$\left|f_{i}\left(u_{1}, u_{2}\right)\right| \leq \frac{1}{1+u_{1}^{2}+u_{2}^{2}}=\Psi\left(\left|u_{1}\right|,\left|u_{2}\right|\right)$.

For $\eta=2$, we get

$\frac{n}{\Gamma(\alpha-1)} \Psi(\eta, \eta)\|g\|_{L^{1}[0,1]} \leq \frac{2}{\Gamma\left(\frac{3}{2}\right)\left(1+2 \eta^{2}\right)}=0.25075 \leq \eta$.

Then, according to the Theorem 9 , the boundary value problem (4.1) has at least one fixed point $u^{*} \in B_{2}$.

Example 2. Consider the following two-dimensional fractional order system

$(4.2)\left(S_{i}\right)=\left\{\begin{aligned} D_{0^{+}}^{\frac{5}{2}} u_{1}(t)=\frac{e^{-t}}{10}\left(u_{1}-u_{2}\right), & D_{0^{+}}^{\frac{5}{2}} u_{2}(t)=\frac{e^{-t}}{10}\left(u_{1}+1\right) \\ u_{1}(0)=0, u_{1}^{\prime}(0)=0, & u_{2}(0)=0, u_{2}^{\prime}(0)=0 \\ a u_{1}(1)-b u_{1}^{\prime}(0)=0, & a u_{2}(1)-b u_{2}^{\prime}(0)=0\end{aligned}\right.$

We have $\alpha=\frac{5}{2}, g(t)=\frac{e^{-t}}{10}, f_{1}\left(u_{1}, u_{2}\right)=\frac{e^{-t}}{10}\left(u_{1}-u_{2}\right), f_{2}\left(u_{1}, u_{2}\right)=$ $\frac{e^{-t}}{10}\left(u_{1}+1\right), f_{i} \in C\left(\mathbf{R}^{2}, \mathbf{R}\right)$, then

$\left|f_{i}\left(x_{1}, x_{2}\right)-f_{i}\left(y_{1}, y_{2}\right)\right| \leq L \sum_{i=1}^{2}\left|x_{i}-y_{i}\right|$

with $L=1$ and $K=\frac{2\left(1-e^{-1}\right)}{10 \Gamma\left(\frac{3}{2}\right)}=0.14265<1$, then hypotheses of

Theorem 8 are satisfied. So, the boundary value problem (4.2) has a unique solution $u \in X$.

Acknowledgement 1. The authors are grateful to the anonymous referees for their valuable comments and suggestions that improved this paper.

\section{References}

[1] R. P. Agarwal, D. O'Regan, S. Stanek, Positive solutions for Dirichlet problems of singular nonlinear fractional differential equations, $J$. Math. Anal.Appl., 371, pp. 57-68, (2010).

[2] B. Ahmad, A. Alsaedi, Existence and uniqueness of solutions for coupled systems of higher-order nonlinear fractional differential equations, Fixed Point Theory Appl., Article ID 364560 (2010). 
[3] B. Ahmad, J. J. Nieto, Riemann-Liouville fractional integrodifferential equations with fractional nonlocal integral boundary conditions, Bound. Value Probl., 36, pp. 1-9, (2011).

[4] B. Ahmad, Nonlinear fractional differential equations with antiperiodic type fractional boundary conditions, Differ. Equ. Dyn. Syst., 21(4), pp. 387-401, (2013)

[5] M. Feng, X. Zhang, W. Ge, New existence results for higher-order nonlinear fractional differential equation with integral boundary conditions, Bound. Value Probl., 2011, Art ID 720-702, (2011).

[6] A. Guezane-Lakoud, R. Khaldi and A. Kılıçman, Solvability of a boundary value problem at resonance, SpringerPlus, (2016) 5:1504.

[7] A. Guezane-Lakoud, A. Ashyralyev, Positive Solutions for a System of Fractional Differential Equations with Nonlocal Integral Boundary Conditions, Differ Equ Dyn Syst, DOI 10.1007/s12591-015-0255-9.

[8] A. A. Kilbas, H. M. Srivastava, J. J. Trujillo, Theory and Applications of Fractional Differential Equations, North-Holland Mathematics Studies, vol. 204. Elsevier, Amsterdam, (2006).

[9] V. Lakshmikantham, A. S. Vatsala, Basic theory of fractional differential equations. Nonlinear Anal., 69, pp. 2677-2682, (2008).

[10] K.Q. Lan, Positive solutions of semi-positone Hammerstein integral equations and applications, Commun. Pure Appl. Anal., 6 (2), pp. 441-451, (2007).

[11] K.Q. Lan, Eigenvalues of semi-positone Hammerstein integral equations and applications to boundary value problems, Nonlinear Anal., $71,(2009)$

[12] S. K. Ntouyas, M. Obaid, A coupled system of fractional differential equations with nonlocal integral boundary conditions, Adv. Differ. Equ., Article ID 130, (2012).

[13] I. Podlubny, Fractional Differential Equations Mathematics in Sciences and Engineering, Academic Press, New York, (1999).

[14] M. Rehman, R. A. Khan, A note on boundary value problems for a coupled system of fractional differential equations, Comput. Math. Appl., 61, pp. 2630-2637, (2011). 
[15] H. Salem, On the existence of continuous solutions for a singular system of nonlinear fractional differential equations, Appl. Math. Comput., 198, pp. 445-452, (2008).

[16] X. Su, Existence of solution of boundary value problem for coupled system of fractional differential equations, Eng. Math., 26, pp. 134137, (2009).

[17] X. Su, Boundary value problem for a coupled system of nonlinear fractional differential equations. Appl. Math. Lett., 22, pp. 64-69, (2009).

\section{A. Guezane-Lakoud}

Laboratory of Advanced Materials

University of Badji Mokhtar Annaba

P. O. Box 12, 23000 Annaba,

Algeria

e-mail : a_guezane@yahoo.fr

\section{G. Rebiai}

Department of Mathematics

University of Guelma

Algeria

e-mail : rebeai-ghania@yahoo.fr,

and

\section{R. Khaldi}

Laboratory of Advanced Materials

University of Badji Mokhtar Annaba

P. O. Box 12, 23000 Annaba,

Algeria

e-mail : rkhadi@yahoo.fr 